\title{
An Experimental Procedure for Autonomous Joint Sensor Estimation Using Adaptive Control
}

\author{
Oliver Astley \\ Vincent Hayward \\ Department of Electrical Engineering and \\ Research Centre for Intelligent Machines \\ McGill University \\ Montréal, Québec, H3A 2A7, Canada \\ oliver@cim.mcgill.ca, hayward@cim.mcgill.ca
}

\begin{abstract}
The autonomous parameter estimation of a manipulator is considered with respect to both dynamic and joint sensor properties. Using methods based on adaptive control, a new formulation is introduced such that bench calibration of the robot joint sensors and actuators is no longer necessary. This method is unique because estimation is done with respect to invariant forces due to gravity loading. The method also guarantees convergence to the true values from arbitrary initial estimates; consequently, the algorithm can also be used for manipulator self test. Experimental results are presented which were performed on two links of a a six degree of freedom hand-controller. Results show that angles can be recovered to an accuracy of $\pm 1^{\circ}$ in the absence of initial estimates.
\end{abstract}

\section{Introduction}

Adaptive Control techniques in robotics have shown powerful results with respect to both overall motion control and identification of robot dynamic properties. These methods however, assume that the robot's joint sensor parameters are known. This paper presents a new method to extract both the dynamic properties and the sensor properties using adaptive control.

For devices which do not have incremental joint encoders to measure joint position, but instead analog sensors, the problem of joint parameter estimation must generally be done by independently measuring joint angles and displacements. Unfortunately, there are many factors which can change these parameters making this manual calibration cumbersome. For this reason we have integrated adaptive control with joint parameter estimation to avoid this manual task.
The methods used in this paper are based on the work done in nonlinear adaptive control by Slotine and Li $[13,15]$. Their methods use the property that constant parameters in the dynamic equations, such as masses, centres of gravity, and inertias of the links, occur linearly with respect to the joint torques; this is known as the regressor form of the robot dynamics. This has been exploited by other researchers in adaptive control including Khosla [6] and Craig [4]. Over the course of their research, Slotine and Li combined their results, which were based on trajectory tracking error, with that of Middleton and Goodwin whose results were based on joint torque error. The combination, known as composite adaptive control, guaranteed both tracking and parameter convergence. It is the guarantee of parameter convergence which is utilised to solve the autonomous estimation problem.

To estimate joint sensor parameters, the robot requires a source of reference. For example, this has been accomplished in the past using a "home position" in which the manipulator is constrained to a known configuration in space. Unfortunately this does not give information concerning the sensor gains. (The conversion factor between the raw sensor output, and the actual angle.) Instead, this paper proposes a technique whereby the manipulator's sensors are estimated using the invariant force of gravity. The gravity terms introduce trigonometric functions within the dynamic model. These terms are replaced by polynomial approximations such that the unknown sensor parameters can be integrated into the composite adaptive control framework.

Section 2 gives a brief summary of Slotine and Li's results in adaptive control. Section 3 presents the new method for joint parameter estimation. Sections 4 and 5 present implementation issues, and an evaluation of 
the experimental results.

\section{Composite Adaptive Control for Robot Joint parameter Estimation}

In this section the results obtained by Slotine and $\mathrm{Li}$ in Composite Adaptive Control are summarised.

\subsection{The Dynamics of a Robot Manipula- tor}

Using $\theta$ as the vector of joint angles, the dynamics of a robot manipulator can be modelled by:

$$
\tau=M(\theta) \ddot{\theta}+C(\theta, \dot{\theta}) \dot{\theta}+G(\theta)
$$

Where $\dot{\theta}$ and $\ddot{\theta}$ are joint velocities and accelerations, $M(\theta)$ is the inertia matrix, $C(\theta, \dot{\theta})$ contains the centrifugal and Coriolis terms, and $G(\theta)$ is the contribution due to gravity. (1) can also be expressed linearly in terms of the physical parameters of the system.

$$
\tau=Y(\theta, \dot{\theta}, \ddot{\theta}) \bar{a}
$$

(2) is known as the regressor form of the dynamics. The vector $\bar{a}$ comprises the unknown parameters of the system; generally this includes, masses, link dimensions, and even constants of friction. It is from the regressor form of the dynamics that composite adaptive control is derived.

\subsection{Composite Adaptive Control}

Composite Adaptive Control evolved from the adaptive control methods of Slotine and Li $[7,10,11,13]$ and Middleton and Goodwin $[8,9]$. These two approaches were combined into composite adaptive control in $[12,14,15]$. The composite adaptive controller uses error measures from both joint tracking and joint torques to update the parameter estimates. The controller requires neither inversion of the inertia matrix or use of joint acceleration measurements.

The control law is:

$$
\tau=\hat{M}(\theta) \ddot{\theta}_{r}+\hat{C}(\theta, \dot{\theta}) \dot{\theta}_{r}+\hat{G}(\theta)-K_{D} s
$$

where $\hat{M}, \hat{C}$, and $\hat{G}$ are estimates of the robot dynamics matrices $M, C$, and $G . K_{D}$ is a linear feedback constant and $s$ is a measure of joint error. The parameter update is defined as:

$$
\dot{\hat{a}}=-P(t)\left(c_{1} Y^{T}\left(\theta, \dot{\theta}, \dot{\theta}_{r}, \ddot{\theta_{r}}\right) s+c_{2} W^{T}(\theta, \dot{\theta}) e\right)
$$

The matrix $Y$ is the regressor form of the robot dynamics and is a function of the both measured joint values, $\theta, \dot{\theta}$, and joint errors restricted to lie on a sliding surface $\dot{\theta}_{r}, \ddot{\theta}_{r}$. W is a filtered form of the regressor matrix $Y$; the filtering is done to avoid measurement of joint acceleration. $e$ is a measure of torque error, $c_{1}$ and $c_{2}$ are weighting constants.

The time varying matrix $P(t)$, in (4), is defined by the Bounded Gain Forgetting method (BGF) [15].

$$
\frac{d}{d t} P^{-1}(t)=-\lambda(t) P^{-1}+W^{T} W
$$

where:

$$
\lambda(t)=\lambda_{0}\left(1-\frac{\|P\|}{k_{0}}\right)
$$

$\lambda_{0}$ and $k_{0}$ are positive constants which define the rate of data forgetting and the upper bound of the $P$ matrix. The product $W^{T} W$ is the solution to a least squares minimization problem of the actual and computed joint torques.

Slotine and Li show that under excitatory conditions the composite adaptive controller attains exponential trajectory convergence and global parameter convergence [15]. This result is important because it can be used as a basis for joint parameter estimation.

\section{Joint Parameter Estimation}

The adaptive control of Slotine and $\mathrm{Li}$ uses the robot dynamic model; this enables prediction of nonlinear robot behaviour. Intuitively, this predictable behaviour should also be utilised in joint parameter estimation schemes in an attempt to achieve an autonomous algorithm.

\subsection{The Problem Caused by Transcen- dentals}

When examining the various forces which are applied to the robot system, gravity stands out as being the most predictable and most reliable of these forces. Using gravity as a reference, it should be possible to find the relationship between joint sensor output and joint angle.

To simplify the derivations and issues involved in joint sensor estimation using adaptive control, a single link rotational manipulator against gravity is used. Its dynamics are:

$$
\tau=m l_{c}^{2} \ddot{\theta}+m l_{c} g \cos (\theta)
$$


where $m$ represents the point mass of the manipulator link, $l_{c}$ is the distance from the joint to the centre of mass, $g$ is the acceleration constant due to gravity, $\theta$ is the joint position, and $\ddot{\theta}$ is joint acceleration. The dynamics in (7) can be rewritten in the regressor form.

$$
[\tau]=\left[\begin{array}{ll}
\ddot{\theta} & g \cos (\theta)
\end{array}\right]\left[\begin{array}{l}
m l_{c}^{2} \\
m l_{c}
\end{array}\right]
$$

The model in (8) is the regressor model which would be used in the standard Slotine and Li composite adaptive controller. Most importantly, (8) assumes the relationship between sensor output and joint angle is known.

Assuming a linear relationship between the joint angle $\theta$, and the sensor output $q$ gives,

$$
\theta=\alpha q+\beta
$$

where $\alpha$ represents the unknown joint gain (Degrees/Volt), and $\beta$ is the unknown joint sensor offset (Degrees). Substituting (9) into (8) gives:

$$
[\tau]=\left[\begin{array}{cc}
\ddot{q} & g \cos (\alpha q+\beta)
\end{array}\right]\left[\begin{array}{c}
\alpha m l_{c}^{2} \\
m l_{c}
\end{array}\right]
$$

From (10) the unknown joint angle parameters of the system $(\alpha, \beta)$ cannot be extracted using the regressor form. The fundamental problem in this formulation is that the transcendental cosine function prevents the $\alpha$ and $\beta$ terms being written linearly with respect to the joint sensor values.

An intuitive solution to this problem is to expand the cosine term in (10) using well known trigonometric expansion formulae. Unfortunately this approach can only isolate the offset term $\beta$. For example expanding the cosine term gives

$$
\begin{aligned}
\cos (\theta) & =\cos (\alpha q+\beta) \\
& =\cos (\alpha q) \cos (\beta)-\sin (\alpha q) \sin (\beta)
\end{aligned}
$$

which does not isolate, in a linear fashion, the $\alpha$ term.

\subsection{Introducing Polynomial Approxima- tions}

It is possible, with arbitrary accuracy, to approximate segments of differentiable nonlinear functions such as cosine and sine functions with polynomials. For example, (12) shows the expansion of $\cos (\theta)$.

$$
\begin{aligned}
\cos (\alpha q+\beta) & =\cos (\theta) \\
& =\underline{a}+\underline{b} q+\underline{c} q^{2}+O^{3}
\end{aligned}
$$

Substituting the cosine approximation in (12) to the regressor form of the single link robot dynamics gives,

$$
[\tau]=\left[\begin{array}{llll}
\ddot{q} & g & g q & g q^{2}
\end{array}\right]\left[\begin{array}{c}
\alpha m l_{c}^{2} \\
a \\
b \\
c
\end{array}\right]
$$

where $a \equiv \underline{a} m l_{c}, b \equiv \underline{b} m l_{c}$, and $c \equiv \underline{c} m l_{c}$.

In (13), the relationship $\tau=Y \bar{a}$ is now in the correct form; all known parameters and variables are in the matrix $Y$, and the unknown parameters are in the parameter vector $\bar{a}$. The condition on parameter and trajectory convergence shown in Section 2 remains unchanged because the transcendental functions are simply being replaced by an equivalent expression. This is valid as long as two conditions are met.

- The order of the polynomial is of sufficient degree to represent the transcendental function it is replacing to a known accuracy.

- The range of operation is not large enough to invoke the periodic properties intrinsic to trigonometric functions.

To find the constants $\alpha$ and $\beta$ from the polynomial coefficients in (13), some simple post processing is required. After estimating the parameters using composite adaptive control for the single link case, and using the manipulator model given in (10), the parameters estimates should approximate:

$$
\begin{aligned}
g m l_{c} \cos (\alpha q+\beta) & =g m l_{c} \cos (\theta) \\
& \approx g\left(a+b q+c q^{2}\right)
\end{aligned}
$$

The constants $\alpha$ and $\beta$ can be found by equating the right side of (14) with (15) such that:

$$
m l_{c} \cos (\theta)=a+b q+c q^{2}
$$

It is necessary to normalise the cosine term by finding the value of $m l_{c}$ in (16). Differentiating the left and right sides of (16) with respect to $\theta$ and $q$ respectively, and equating to zero gives.

$$
-m l_{c} \sin (\theta)=b+2 c q=0
$$

Assigning $q^{*}$ to the solution of (17) enables $m l_{c}$ to be found by:

$$
m l_{c} \cos \left(\theta^{*}\right)=m l_{c}=a+b q^{*}+c q^{* 2}
$$

Therefore by finding the maximum point on the cosine approximation, the value for $m l_{c}$ can be found. 
This allows the fitting of $\cos (\alpha q+\beta)$ to be independent of the mass properties of the manipulator according to:

$$
\cos (\theta)=\cos (\alpha+\beta)=\frac{a+b q+c q^{2}}{m l_{c}}
$$

Using (19), the value of $\cos (\theta)$ can be found for any value of sensor measurement $q$. Rearranging (19) to isolate the $\alpha$ and $\beta$ constants yields:

$$
\theta=\alpha q+\beta=\arccos \left(\frac{a+b q+c q^{2}}{m l_{c}}\right)
$$

To solve for $\alpha$ and $\beta$ in (20) a curve fitting strategy is used; the experimental work presented in Section 5 uses a least squares technique.

\subsection{The Multi-Link Case}

Unfortunately it is not possible to substitute all transcendental functions with polynomials in the complete robot dynamics for two reasons.

- The columns of the regressor matrix will become rank deficient. Therefore there will not be a unique solution and parameter convergence will not be guaranteed.

- For every transcendental function, one unknown parameter gets substituted for at least three new ones, causing a rapid explosion in the number of parameters. (Processing time increases with the square of the number of unknown parameters.)

Therefore it is proposed that the gain parameters be found separately from the offset parameters. Each $\alpha$ term is found individually by locking all joints except the joint to be estimated. This has the effect of making the robot into a single link mechanism and greatly decreases the complexity of the dynamics. By using composite adaptive control methods on the single link equivalent, a polynomial approximation can then be used to find $\alpha$ and $\beta$. Only the $\alpha$ term will be valid because the displacement of the centre of mass per sensor unit will be invariant to the location of the centre of mass for the one link system; the offset $\beta$, however, will be taken with respect to direction of gravity not the preceding link. Once all the gain terms have been found, the offset terms can be extracted using trigonometric properties, as in (11).

\section{Experimental Procedure}

The algorithm is tested on two links of a 6 degree of freedom hand controller [5]. The joints are actuated through tendons which are connected to two motors working in a pull-pull configuration. The joint sensor is a differential LED-receiver system which produces a current proportional to the joint position. After signal conditioning circuitry, the sensor output is a voltage with a range of approximately \pm 8 volts. The velocity reading is obtained from an operational amplifier in a differentiating configuration. These signals are fed into a 16 bit analog to digital converter which is connected to a standard $486 \mathrm{PC}$ running at $66 \mathrm{MHz}$. The sampling rate is $1 \mathrm{kHz}$.

The assumptions made for algorithm implementation are:

- The joint limits are known with respect to the sensor voltage output.

- The sign of the torque applied, position (in sensor units) and velocity are all uniform to prevent positive feedback in the linear PD feedback of the controller.

- The structure of the robot is known.

Experiments on the device using the algorithm exposed several factors necessary for successful implementation. These include, trajectories, friction, and sensor scaling.

\subsection{Trajectories}

There has been much work in finding suitable excitatory trajectories for adaptive control, [1-3]. Most of this research, however, has been with respect to linear systems. The approach we took was to use a frequency rich trajectory to excite as many modes of the system as possible. This can be achieved theoretically by applying a train of impulses or applying white noise to the system. In experiments, it was found that a triangular wave trajectory gave the best parameter convergence results. Although a rigorous explanation is not available to substantiate this finding, it is possible, in hindsight, to hypothesise on the intuitive factors which contribute to this result.

A mechanical system can be approximated to a double integrator, such that a force is applied to the input and the output is a displacement. Since twice differentiating a triangular wave trajectory gives an acceleration profile made up of impulses, full frequency content is experienced at the force input to the system. This explanation is easily understood from an intuitive level; however, a more rigorous explanation is beyond the scope of this paper. 


\subsection{Friction}

Friction effects can be included in the original adaptive controller; this is not the case, however, for the polynomial approximation. This can be attributed to the lack of structure in a polynomial when compared to the original transcendental function. (i.e. the polynomial can take many forms but the cosine structure is fixed.) For this reason, adding friction models to the adaptive control dynamic model when using the polynomial approximation is detrimental. Instead, a feed-forward Coulombic friction term was augmented to system outside of the adaptive controller. The coefficient of Coulombic friction was found autonomously using a closed loop trajectory under PD control.

\subsection{Scaling}

The most volatile period of the adaptive control estimation is the initial one or two seconds after start up. This is because the initial parameter estimates, in the manipulator model, are all zero. To minimize this volatility it was found beneficial to scale the sensor output voltages to between \pm 1 Volt. This prevents polynomial terms of the form $q_{i}^{n}$ becoming unbounded such that:

$$
\left\|q_{i}\right\| \leq 1 \Longrightarrow\left\|q_{i}^{n}\right\| \leq 1 \quad \forall n>0
$$

\section{$5 \quad$ Experimental Results}

To estimate the joint sensor parameters $\alpha$ and $\beta$ for the two link mechanism, a two phase procedure is required. First the $\alpha$ (gain) terms are estimated for each joint independently, then the $\beta$ (offset) terms are found together. Figure 1 shows the convergence of polynomial parameters for the adaptive control estimation of the joint $2 \alpha$ term. Parameters $a, b$, and $c$, on the graph correspond to the model in (13). The graph shows that the polynomial coefficients are estimated within approximately 15 seconds.

To evaluate the accuracy and consistency of the algorithm, the adaptive control estimation was performed over ten trials. Table 1 shows the results of the complete estimation over these trials.

The results show that the algorithm has an accuracy of about \pm 1 degree when all factors are considered. This was achieved using arbitrary initial parameter estimates. What is more, the algorithm also estimates the dynamic parameters of the system, we used this data to perform gravity compensation on the hand-controller.

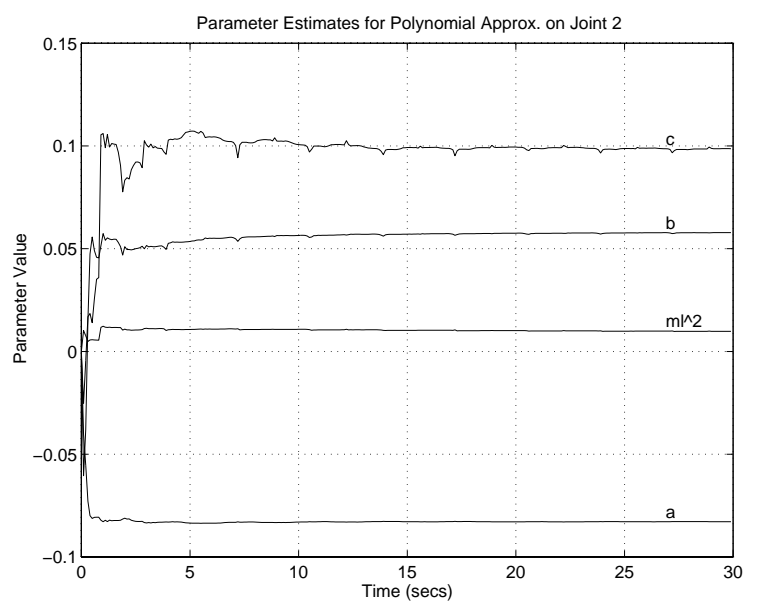

Figure 1: Parameter Estimate Evolution for Polynomial Approximation on Joint 2

\section{Conclusion}

The algorithm presented in this paper enables autonomous joint and dynamic parameter estimation of a multiple link robot. The estimation is performed on-line using adaptive control methods introduced by Slotine and Li. Their adaptive control method is advantageous for estimation because it is globally convergent with respect to both trajectory tracking and parameter convergence. This implies that a priori knowledge of the system parameters is not required to achieve system parameter convergence; the Slotine and $\mathrm{Li}$ method, however, requires that the manipulator's joint parameters are estimated before operation. Experimental results are presented for joint parameter estimation of a two link planar robot; the results show an accuracy of about $\pm 1^{\circ}$.

Our algorithm requires only knowledge of the manipulator dynamic model structure and does not require a priori joint sensor information other than assuming the joint sensor is linear. This means that after switching the power to the robot on, the robot can autonomously find its dynamic and joint sensor properties without human interaction, use of specialised measuring equipment, or physical constraints on the robot.

Presently the algorithm is being used for both self calibration and gravity compensation of a 6 dof handcontroller (The original purpose of this research.) The algorithm naturally lends itself to these applications because both dynamic and joint sensor parameters are found upon completion. Also, this algorithm is useful for performing a manipulator self test. In the event of a sensor or actuator degradation leading to failure, the 


\begin{tabular}{|c|c|c|c|c|}
\hline & $\begin{array}{c}\alpha_{1} \\
\text { degrees/Volt }\end{array}$ & $\begin{array}{c}\beta_{1} \\
\text { degrees }\end{array}$ & $\begin{array}{c}\alpha_{2} \\
\text { degrees/Volt }\end{array}$ & $\begin{array}{c}\beta_{2} \\
\text { degrees }\end{array}$ \\
\hline Manual Calibration & -7.638 & -17.544 & -9.052 & 26.299 \\
\hline Mean A-C Estimation & -7.465 & -17.841 & -8.537 & 26.673 \\
\hline Maximum A-C Estimation & -6.963 & -16.890 & -8.322 & 27.250 \\
\hline Minimum A-C Estimation & -8.164 & -18.115 & -8.795 & 25.273 \\
\hline Standard Deviation & 0.465 & 0.421 & 0.161 & 0.615 \\
\hline
\end{tabular}

Table 1: Consistency of Adaptive Control Robot Parameter Estimation (Over 10 experiments)

algorithm will converge erratically or fail to converge altogether. This lack of convergence can be used to isolate the hardware problem. Currently work in sensor and structural failure detection is on going.

\section{Acknowledgements}

Funding for this project has been provided by the "Haptic Devices for Teleoperation and Virtual Environments" (AMD-5) funded by IRIS, the Institute for Robotics and Intelligent Systems part of Canada's National Centres of Excellence program (NCE). Additional funding was provided by NSERC, the National Science and Engineering Council of Canada, in the form of a postgraduate scholarship for the first author and an operating grant for the second. The first author also received funding from a Raychem Cooperation Paul M. Cook International Scholarship.

\section{References}

[1] B. Armstrong. On finding 'exciting' trajectories for identification experiments involving systems with nonlinear dynamics. IEEE International Conference on Robotics and Automation, 1987.

[2] S. Boyd and S. Sastry. On parameter convergence in adaptive control. System and Control Letters, 3:311-319, December 1983.

[3] S. Boyd and S. Sastry. Necessary and sufficient conditions for parameter convergence in adaptive control. Automatica, 22(6):629-639, 1986.

[4] J.J. Craig, P. Hsu, and S.S. Sastry. Adaptive control of mechanical manipulators. The International Journal of Robotics Research, 6(2), 1987.

[5] V. Hayward. Toward a seven axis haptic interface. IROS'95, Int. Workshop on Intelligent
Robots and Systems, Pittsbugh, PA,, 3:134-140, August 1995.

[6] K.K. Khosla and T. Kanade. Parameter identification of robot dynamics. Proceedings of the 24th Conference on Decision and Control, pages 1754-1760, 1985.

[7] W. Li and J-J.E. Slotine. Indirect adaptive robot control. Proceedings of the IEEE International Conference on Robotics and Automation, pages 704-709, 1988.

[8] R.H. Middleton. Hybrid adaptive control of robot manipulators. Proceedings IEEE Conference on Decision and Control, 2:1592-1597, 1988.

[9] R.H. Middleton and G.C. Goodwin. Adaptive computed torque control, for rigid link manipulators. 25th I.E.E.E. Conference on Decision and Control, 1989.

[10] J.J.E. Slotine and W. Li. On the adaptive control of robot manipulators. A.S.M.E. Winter Annual Meeting, Anaheim, CA, 1986.

[11] J.J.E. Slotine and W. Li. Adaptive manipulator control: A case study. IEEE International Conference on Robotics and Automation, 3:1392-400, 1987.

[12] J.J.E. Slotine and W. Li. Adaptive robot control: A new perspective. Proceedings IEEE Conference on Decision and Control, 1:192-198, 1987.

[13] J.J.E. Slotine and W. Li. On the adaptive control of robot manipulators. The International Journal of Robotics Research, 6(3), 1987.

[14] J.J.E. Slotine and W. Li. Adaptive robot control: A case study. IEEE Transactions on Automatic Control, 33:995-1003, 1988.

[15] J.J.E. Slotine and W. Li. Composite adaptive control of robot manipulators. Automatica, 1:509-519, 1989. 